\title{
H.761 Support of a DOM Level 4 Core specification in NCLua scripts
}

\author{
Alan L. V. Guedes \\ TeleMídia - PUC-Rio \\ alan@telemidia.puc-rio.br
}

\begin{abstract}
Web developers commonly run HTML template engines at the client-side using JavaScript. One upper hand is to give more development flexibility comparing to the server-side. In this way, we may cite web frameworks webcomponents, ReactJS, Vue, and others. Those frameworks use the DOM API to access the template data inserted in the HTML document. Then the same DOM API is used to insert more HTML code. Currently, NCL not support access to the current document. But it can modify the document by NCL Editing commands in NCLua scripts. We propose NCLua scripts partially support the DOM Code API.
\end{abstract}

\section{KEYWORDS}

NCL, Ginga

\section{BACKGROUND}

Web developers commonly run HTML template engines at the client-side using JavaScript. One upper hand is to give more development flexibility comparing to the server-side. In this way, we may cite web frameworks webcomponents, ReactJS, Vue, and others. Those frameworks use the DOM API to access the template data inserted in the HTML document. Then the same DOM API is used to insert more HTML code.

Currently, H.761 NCL[1] do not support access to the current document. But it can modify the document by NCL Editing commands in NCLua scripts.

\section{PROPOSAL}

We propose NCLua scripts partially support the DOM API. More precisely, we propose the NCLua support the document object from the DOM Core API. We details our document object in what follows. This element is based on the lua-gumbo ${ }^{1}$. Its structure and API of this tree mostly follows the DOM Level 4 Core specification ${ }^{2}$.

Properties:

- documentElement: The root Element of the document (i.e. the $<$ html $>$ element).

- head: The <head $>$ Element of the document.

- body: The <body> Element of the document.

- title: A string containing the document's title (initially, the text contents of the $<$ title $>$ element in the document markup).

\footnotetext{
${ }^{1}$ https://craigbarnes.gitlab.io/lua-gumbo/\#document
}

${ }^{2}$ https://dom.spec.whatwg.org/

In: Future of Interactive Television Workshop (V WTVDI), Rio de Janeiro, Brasil. Anais Estendidos do Simpósio Brasileiro de Sistemas Multimídia e Web (WebMedia). Porto Alegre: Sociedade Brasileira de Computação, 2019.

ISSN 2596-1683

\author{
Sergio Colcher \\ Informatics Departament - PUC-Rio \\ colcher@inf.puc-rio.br \\ - forms: An ElementList of all < form> elements in the docu- \\ ment. \\ - images: An ElementList of all <img> elements in the docu- \\ ment. \\ Methods: \\ - getElementById(elementId): Returns the first Element node \\ in the tree whose id property is equal to the elementId string. \\ - getElementsByTagName(tagName): Returns an ElementList \\ containing every child Element node whose locaName is \\ equal to the given tagName argument. \\ - getElementsByClassName(classNames): Returns an Ele- \\ mentList containing every child Element node that has all \\ of the given class names. Multiple class names can be spec- \\ ified by passing a string with several names separated by \\ whitespace.
}

\section{USE CASE}

The following code uses the getElementById to get a media object. Then it uses an NCLua command to add a property to it at runtime.

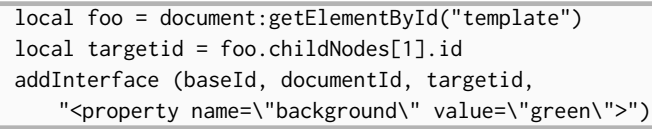

Listing 1: NCL code fragment using the proposed approach.

\section{REFERENCES}

[1] ITU. 2009. H.761: Nested Context Language (NCL) and Ginga-NCL for IPTV Services. Technical Report. ITU, Geneva, Switzerland. https://www.itu.int/rec/T-REC-H. 761 